\title{
"E la mia vita è divina”. Suggestioni orientali nel Meriggio dannunziano
}

\section{“And my life is divine”. Oriental suggestions in Meriggio by D’Annunzio}

\author{
Paolo TABacchini [tabacchini.p@gmail.com] \\ Univerzita Palackého v Olomouci, Repubblica Ceca
}

\begin{abstract}
Lo scopo dell'articolo è quello di mettere in evidenza un aspetto peculiare della spiritualità di D’Annunzio: la concordanza con elementi della tradizione indiana nel sentire e concepire la spiritualità divina e umana. Dopo un'introduzione al tema che illustra lo stato della critica sull'argomento e mostra le maggiori "prove" testuali ed extra-testuali della conoscenza e dell'attenzione dell'autore alla cultura indiana, l'articolo si concentra sul commento e sull'interpretazione della composizione Meriggio, opera che rappresenta una delle vette, oltre che della sua produzione letteraria, anche del processo di sincretismo tra il suo sentimento spirituale e la religiosità di ascendenza indiana.
\end{abstract}

\section{Parole Chiave}

Gabriele D’Annunzio; Meriggio; Spiritualità; Induismo; Culture orientali

\begin{abstract}
The purpose of the article is to highlight a peculiar aspect of D'Annunzio's spirituality, such as the concordance with elements of the Indian tradition in feeling and conceiving divine and human spirituality. The introduction to the topic, primarily illustrates the state of the arts on the subject and shows the major textual and extra-textual "evidence" of the author's knowledge and attention to Indian culture. Then, the article focuses on the commentary and interpretation of Meriggio, the work which represents one of the peaks - as well as of his literary production - of the process of syncretism between the spiritual feeling of the author and the religiosity of Indian ancestry.
\end{abstract}

\section{KEYWORDS}

Gabriele D’Annunzio; Meriggio; Spirituality; Hinduism; Oriental cultures

RICEVUTO 2021-03-16; ACCETTATO 2021-08-06 


\section{Un problema critico: la spiritualità dannunziana}

Partiamo da un problema critico: Gabriele D’Annunzio è una delle figure della tradizione letteraria italiana sulla quale la critica ha speso molto inchiostro ma al contempo si è spesso cristallizzata su poli antitetici, conferendo un'immagine spesso stereotipata dell'autore. In questo processo di polarizzazione della critica dannunziana, va da sé che il primo della lista è proprio D’Annunzio, impegnato per tutta la vita a definire molte immagini di sé, spesso antitetiche. Il D’Annunzio poeta-vate, l'arbiter elegantiarum, il condottiero della patria mutilata, il D'Annunzio dalla vita inimitabile; ma anche il poeta notturno, il malinconico spirito della decadenza, il genio tentato di morire. Tanti volti, o tante maschere, corrispondenti anche a diversi modelli che via via la critica ha privilegiato, conferendo al personaggio immagini differenti e parziali, spesso molto simili a stereotipi (Ferrara 1981a: 67 e 74).

Una questione che si è prestata e si presta tuttora a notevoli speculazioni è quella relativa alla spiritualità dannunziana. È già nei primissimi anni del Novecento, quando il poeta abruzzese era ancora nel pieno della sua sperimentazione poetica, che prende avvio la diatriba tra due opposti modelli critici: da una parte, il D’Annunzio tutto materia e sensualità, l'esteta gelido, il sofista dell'eros; dall'altra l'altro D'Annunzio, quello tutto spirito, il sacerdote di Pan sulla terra (Costa 1988; Mazza 1995; e Crotti 2016).

I prodromi di queste due immagini dannunziane sono da rintracciarsi nella diatriba tra Giuseppe Antonio Borgese e Benedetto Croce (Borgese 1903; Croce 1904; e Borgese 1909). In uno scritto del 1903, Borgese sottolinea la relazione tra i sentimenti spirituali del poeta e la tradizione religiosa indiana, sostenendo ad esempio che «[i]l Pan di Gabriele d'Annunzio è collegato col Brahma o Atman degli Indiani» (Mazza 1995: 79). Croce risponderà l'anno seguente, negando qualsiasi dimensione spirituale in D’Annunzio, men che meno di estrazione orientale. Infatti, riguardo al confronto tra «l'unità panica dannunziana» e la «concezione indiana», quest'ultimo sostenne che la prima «non è vita superiore [...] ma vita bassa e primitiva» (Mazza 1995: 78 nota 78). Il veto crociano farà cambiare idea al primo (Borgese 1909), che aderirà alla tesi del filosofo, tacciando D’Annunzio di «franco materialismo» (Turoff 2017: 454). Per una più ampia disanima della polemica, si vedano Turoff (1989: 311), Mazza (1995: 78-79) e Turoff (2017: 454).

Come si può notare, l'origine delle due posizioni della critica dannunziana sul tema coincidono anche con la genesi della questione delle influenze induiste sul pensiero spirituale di D’Annunzio (Turoff 1989: 311-312). Ora sminuite, ora aumentate di volume, le suggestioni della cultura indiana, come del resto quelle di un più generico orientalismo del poeta, hanno avuto varie manifestazioni nella critica dannunziana (Ferrara 1981a; e Ferrara 1981b).

\section{D’Annunzio e la cultura orientale}

Se non si può parlare, certamente, di una spiritualità istituzionalizzata, o di una vera e propria religiosità di D’Annunzio, è altrettanto innegabile che la dimensione dello spirito abbia comunque un ruolo, se non centrale, quantomeno notevole, nella sua esperienza poetica. Molte sono, infatti, le testimonianze di una sua apertura verso l'infinito, verso la misticità, colta ora nella 
natura, ora nei sentimenti sperimentati dal poeta nella sua intimità (Ferrara 1981a e b; Mazza 1995; Burrini 2004; Zollino 2013; Turoff 2017).

In questa sensibilità per l'infinito, in questo senso del divino, è anche visibile una ispirazione che trae spunto da culture lontane. La tradizione occidentale è presente e ben visibile nel poeta abruzzese. Il sostrato composito, tipicamente europeo, frutto del remoto sincretismo tra tradizione classica (greco-latina) e cristiana, è certamente presente nel suo immaginario poetico. Tuttavia, esiste anche una linea più sottile, più evanescente, che percorre l'intera produzione dannunziana: la cultura orientale, in particolar modo la tradizione sapienziale indiana, con la sua millenaria storia, è una suggestione alla quale D’Annunzio soggiace e che ricerca ed esplora con vivace spirito pionieristico considerando l'altezza cronologica nella quale si trovò a vivere.

Numerose e variegate sono le prove che testimoniano l'attenzione del poeta nei riguardi della cultura indiana. È un'attrazione, questa, figlia anche, certamente, del gusto per l'Oriente tipico di molti intellettuali della sua generazione e di quelle immediatamente precedenti. Si pensi, infatti, all'amore per il primitivo remoto, manifestato da alcuni intellettuali romantici, come il Goethe del West-östlicher Divan, e post-romantici, come Théophile Gautier. Inoltre, è proprio tra Otto e Novecento che prende avvio lo studio, tanto antropologico quanto linguistico, della cultura indiana, il quale produrrà eccellenti risultati nella mitologia comparata e nell'indoeuropeistica (si veda per esempio l'attività di Georges Dumézil). Anche nella poesia del secondo '800, la splendeur oriental esercita un'attrazione magnetica di forza notevole alla quale molti autori soggiacciono.

D'Annunzio, indubbiamente, entra in piena risonanza con questa fascinazione per l'Oriente che investe l'Europa in quegli anni. Tuttavia, in lui il rimando all'Oriente indiano non è solo un semplice ornamento, né una suggestione meramente intellettuale. D’Annunzio sembra invece trovare nella cultura indiana un contrappunto perfetto al suo modo di sentire e concepire la dimensione spirituale della vita. Quello che è stato giustamente definito come estetismo mistico di D’Annunzio (Turoff 2017) trova un pieno accordo con la concezione filosofico-religiosa dell'Induismo e da essa trae forza concettuale. Inoltre, tale prospettiva esistenziale è anche il fulcro ove si innerva la sua intera esperienza poetica. In tutta l'attività poetica di D'Annunzio, da Primo vere al Libro segreto, è visibile lo sforzo immane di cogliere quel «prodigio» (Turoff 2017: 459-461) che è lo spirito nella materia, di vedere il Tutto, l'Eterno, trascendendo il contingente. È dunque un fine spirituale quello che anima la poesia di D'Annunzio, la quale però non lascia mai, per così dire, il fenomenico, il materiale. Quella di D’Annunzio è una ricerca dello spirituale, del divino, tutta concentrata sull'immanenza della materia, sul mondo fenomenico.

Il concetto di estetismo mistico (Turoff 2017: 453) riduce la annosa e vexata quaestio, che divide in due filoni la critica dannunziana, ad una mera questione prospettica. In D'Annunzio, dunque, animato com'è da una spiritualità tutta immanente alla materia, convivono due tensioni contemporanee: verso la materia e al di là della materia.

Scopo di questo articolo è mostrare le caratteristiche della spiritualità dannunziana attraverso l'analisi critica e l'interpretazione di Meriggio, luogo in cui questa raggiunge una delle sue massime espressioni, in accordo con il contesto altrettanto speciale di Alcyone. Pertanto, procediamo con ordine. Prima di affrontare il testo, è necessario illustrare il contesto in cui sorge questo particolare approccio spirituale.

La «prove» della conoscenza e dell'interesse, per nulla di secondaria importanza nella vita intellettuale del poeta, a cui si è alluso in precedenza, sono numerose e di varia natura e hanno 
inoltre una genesi molto precoce. Il primo testimone è legato alla vita amorosa di D’Annunzio, ovvero una lettera datata 11 ottobre 1881 e indirizzata a Giselda Zucconi (e conservata nell'Archivio personale della Fondazione Il Vittoriale degli Italiani, cfr. Turoff 2017: 455-456), ossia l'Elda o Lalla dell'epistolario, primo amore dal poeta, alla quale l'anno seguente dedicherà la sua seconda raccolta di liriche, Canto novo. Nella lettera, D’Annunzio allude al Rāmāyana di Vālmìki, monumento della poesia e della cultura religiosa indiana nel quale, in tono epico e mitografico, si narrano le imprese del principe Rāma, settimo avatāra di Viṣnu. Il mito indiano viene utilizzato dal poeta per narrare il rapporto tra lui ed Elda, in un processo di identificazione simmetrica tra figure letterarie e le figure reali (Rāma : Sītā = D’Annunzio : Giselda).

La seconda prova è un articolo scritto da D'Annunzio dietro lo pseudonimo (anch'esso orientaleggiante) di Shiun-Sui-Katsu-Kava dal titolo Toung-Hoa-Lou. Ossia Cronica del Fiore dell'Oriente, pubblicato in «Tribuna» il primo dicembre 1884 (Mazza 1995: 78 e nota 76). Nell'articolo, il poeta fa una suggestiva comparazione tra l'iconografia sacra e la ritualità cristiana e buddista che mostra la frequentazione delle opere d'arte afferenti a quella tradizione religiosa (per un approfondimento su una tematica affine, ossia l'influenza della cultura giapponese in D'Annunzio, si vedano Basile 1983; Turoff 2005; Leo 2019).

Altra prova è un noto brano de Il piacere (libro II, cap. 1, edizione di riferimento: D’Annunzio 1989) nel quale un convalescente Sperelli medita sulle Upaniṣad, una collezione di testi sapienziali capitale del canone induista, sulla scorta ovviamente dalla rilettura schopenhaueriana (Turoff 2017: 462). Qui cita addirittura la massima in sanscrito: Tat tvam asi 'Questa cosa [riferito all'Ātman-Brahman], sei tu', terza delle quattro Mahāvākyas 'Grandi parole', le quali sintetizzano i pilastri della spiritualità induista (Ferrara 1981a: 70).

La quarta prova è l'amicizia profonda con Angelo Conti, il quale, con le parole di Mario Guabello, fu un: «buddista propagatore del verbo di Schopenhauer» (Turoff 2017: 464). I due si incontrarono per la prima volta già nel 1882 e tra loro nacque un sodalizio intellettuale saldo e duraturo.

Queste sono alcune delle testimonianze più stringenti. Ad esse si potrebbero aggiungere le numerose allusioni a una concezione dello spirito affine in maniera notevole alla tradizione indiana e che investono l'intera produzione poetica dannunziana. Per una disamina completa e puntuale di tali controprove, si rimanda al lavoro pregevole della prof.ssa Barbara Turoff del Pratt Institute di New York (USA), la quale ha dedicato al tema una parte considerevole della sua attività di ricerca, a partire dalla sua tesi di dottorato (Turoff 1990).

Sempre grazie all'impegno della prof.ssa Turoff, si ha un ulteriore e solido teste (Turoff 1989): la compilazione di un catalogo ragionato delle opere afferenti alla cultura orientale nella biblioteca personale di D’Annunzio, conservate nel museo della Fondazione Il Vittoriale degli Italiani. Solo quelli relativi alla cultura indiana (religione, filosofia, arte, letteratura, ecc.) sono più di 70 volumi, dei quali molti recano postille e note autografe di D’Annunzio (Turoff 1989: 312; Turoff 2017: 466). I dati quantitativi (numero di volumi) e qualitativi (varietà di temi e annotazioni) messi in evidenza dalla ricerca documentaria e critica della prof.ssa Turoff, dimostrano un'attenzione preponderante, si direbbe quasi ossessiva, del poeta nei riguardi della cultura indiana.

Sempre all'interno dell'Archivio personale dannunziano (presso la Fondazione Il Vittoriale degli Italiani) vi è anche un manoscritto recante un Sutra presente dell'Abhidharma pițaka, terza sezione del canone buddista in lingua pāli, avente come argomento la «vacuità dei sensi» (Mazza 1995: 78 e nota 75). 
Nella collezione delle opere d'arte dannunziane conservata al Vittoriale vi sono, inoltre, moltissimi esemplari dell'arte orientale, in modo particolare relativi all'arte sacra buddista, che manifestano una singolare passione verso quella tradizione. Ad essa è stata dedicata anche la mostra Racconti d'Oriente, a cura di Alessandra Coppa (Project manager Sabina Antonini, EN SPACE network) allestita dal 10/05/2015 al 31/10/2015 presso il Museo D’Annunzio segreto (sempre nel complesso del Vittoriale).

Le «prove» sin qui esposte, dunque, mostrano chiaramente uno spiccato e dichiarato interesse di D’Annunzio nei riguardi della cultura indiana, che ha un'origine remota e verrà sempre più approfondito negli anni, entrando prepotentemente nel suo modo di sentire e di conseguenza nella sua poesia.

Snodo centrale ed eccezionale di questa sintesi tra il sentire dannunziano e la filosofia induista è la raccolta Alcyone. Il contesto in cui nascono le liriche che confluiranno nella raccolta del 1903, sono del tutto singolari: quattro estati di «ritiro» presso la costiera toscana, in Versilia, con la Duse (sulla raccolta si vedano ad es. Gibellini 1985; Burrini 2004; e Roncoroni 2020).

Come già sottolineato da Mario Ferrara (Ferrara 1981a: 69; Turoff 2017: 465), in questa raccolta, e in particolare nella "perfetta espressione» rappresentata da Meriggio, si «traduce e realizza in piena concretezza lirica» l'intuizione induista del Tat tvam asi. La cornice di Alcyone, quindi, è quella del ritiro, nient'affatto erotico e ozioso, ma piuttosto contemplativo, spirituale (sul componimento, oltre agli appena citati, si vedano anche Zollino 2013 e Roncoroni 2020; sul motivo del meriggio, cfr. Giusti 2005).

\section{Commento e interpretazione di Meriggio}

Veniamo ora al testo preso in esame, Meriggio (D’Annunzio 2020: 299-309), del quale si offrirà innanzitutto un commento analitico (suddiviso nelle quattro strofe che costituiscono il componimento), al quale seguirà una sintesi interpretativa.

\section{Commento}

Ecco la prima strofa:

A mezzo il giorno

sul Mare etrusco

pallido verdicante

come il dissepolto

bronzo dagli ipogei, grava

la bonaccia. Non bava

di vento intorno

alita. Non trema canna

su la solitaria

spiaggia aspra di rusco,

di ginepri arsi. Non suona

voce, se ascolto.

Riga di vele in panna 
verso Livorno

biancica. Pel chiaro

15

silenzio il Capo Corvo

l'isola del Faro

scorgo; e più lontane,

forme d'aria nell'aria,

l'isole del tuo sdegno,

o padre Dante,

la Capraia e la Gorgona.

Marmorea corona

di minaccevoli punte,

le grandi Alpi Apuane

regnano il regno amaro,

dal loro orgoglio assunte.

Il componimento prende avvio dall'evocazione del contesto nel quale si realizzerà l'evento estatico (il quale, come si vedrà, sarà il tema dell'ultima e in parte della penultima strofa). Quando e dove siamo? Lo dice subito nei primissimi versi (cfr. vv. 1-2: «a mezzo il giorno / sul Mare etrusco»): è mezzogiorno (come del resto suggerisce anche il titolo, con un termine più aulico), su una spiaggia tirrenica (cfr. v. 2: «Mare etrusco»).

Da subito però vi è un carattere che sarà presente per tutto il componimento: le cose rappresentate sono sé stesse, ma simboleggiano anche altro. L'ambiente designato è connotato con attributi chiari e reiterati che suscitano l'immagine mentale della stasi, dell'inerzia, del totale abbandono all'impasse, tanto del corpo quanto dello spirito (cfr. vv. 4-6: «come il dissepolto / bronzo degli ipogei grava / la bonaccia [...]»). La sonnolenza e la vaghezza sono i correlativi emotivi degli oggetti fenomenici, sia naturali che artificiali, che vengono evocati. Segue un'ulteriore specificazione di questa inerzia che stagna attorno, scandita da tre negazioni (cfr. v. 6: «[...] Non bava», v. 8: «[...] Non trema [...]», v. 11: «[...] Non suona»); e ancora, una nave in panne, un'aria diafana (cfr. vv. 13-15), «pel chiaro / silenzio» (quel «pel» del v. 15 introduce un moto per luogo) attraverso la quale si intravede il capo di una terza isola (v. 17: «del Faro»).

C'è un gusto per la precisione che si nota nella denominazione dei luoghi geografici e che ricollega il brano allo stile dantesco della Commedia (la quale verrà evocata anche direttamente, $\mathrm{e}$ proprio in un contesto geografico, poco dopo). Tuttavia, come si vedrà, l'applicazione di questo espediente stilistico porterà ad un fine poetico differente.

Le terre indicate ai versi 20-22 («l'isole del tuo sdegno, / o padre Dante, / la Capraia e la Gorgona») evocano l'eco del tragico canto di Ugolino (Commedia, Inf. XXXIII, vv. 79-87; qui ed altrove, l'edizione di riferimento è Dante 1994):

\footnotetext{
Ahi Pisa, vituperio de le genti

del bel paese là dove 'l sì suona,

poi che i vicini a te punir son lenti,

muovasi la Capraia e la Gorgona,

e faccian siepe ad Arno in su la foce,
} 
sì ch'elli annieghi in te ogne persona!

84

Che se 'l conte Ugolino aveva voce

d'aver tradita te de le castella,

non dovei tu i figliuoi porre a tal croce.
87

dove, nella vibrante invettiva contro Pisa, Dante sprona le stesse isole (in un parossismo estremo di violenza) ad intervenire, vista la pigrizia dei vicini (cfr. Inf. XXXIII, v. 81: "poi che i vicini a te punir son lenti»), e a punire l'ingiustizia del Comune che ha inflitto una così crudele e insensata condanna al Conte.

Queste terre, benché accuratamente individuate sia nello spazio (si veda il termine geografico «isole» e i due toponimi esatti «Capraia» e «Gorgona»), che nel loro significato storico-culturale all'interno della tradizione letteraria italiana (cfr. v. 21: «o padre Dante»), sono colte nella loro evanescenza di fenomeni illusori (cfr. v. 19: «forme d'aria nell'aria»). Veniamo ora alla seconda strofa:

La foce è come salso stagno. Del marin colore, per mezzo alle capanne,

per entro alle reti

che pendono dalla croce

degli staggi, si tace.

Come il bronzo sepolcrale

pallida verdica in pace

quella che sorridea.

Quasi letèa,

obliviosa, eguale,

segno non mostra

di corrente, non ruga

d'aura. La fuga

delle due rive

si chiude come in un cerchio

di canne, che circonscrive

l'oblìo silente; e le canne

non han susurri. Più foschi

i boschi di San Rossore

fan di sé cupa chiostra;

ma i più lontani,

verso il Gombo, verso il Serchio,

son quasi azzurri.

Dormono i Monti Pisani

coperti da inerti

cumuli di vapore. 
Nella seconda strofa è ancora protagonista la natura, il contesto, che però tende ora a circoscrivere un paesaggio sempre più dell'animo, dell'io, piuttosto che dell'ambiente. Se nella prima strofa si è infatti insistito sulle immagini mentali ed i correlativi oggettivi della stasi e della vaghezza, qui si sottolinea invece il concetto di limite, di confine entro il quale l'evento «mistico» si determinerà. Già dall'inizio, si designa un chiaro limitante, «la foce», che oltre ad essere un altro tecnicismo geografico, evoca l'immagine della fine, del limite, la quale «è come salso / stagno» (vv. 28-29), quindi un delimitato e chiuso specchio d'acqua. L'aggettivo «sepolcrale» (v. 34) evoca ancora, insieme, il silenzio e la morte, la fine per eccellenza. Il trittico «chiude» (v. 43), «circonscrive» (v. 44) e «chiostra» (v. 48) sottolineano di nuovo il motivo della chiusura, del confine (che si ricollega a sua volta al limite rappresentato della siepe leopardiana, cfr. Zollino 2013). Nei versi 37-42 si insiste nuovamente sui temi dello stordimento, dello smarrimento dei sensi e della stasi, nei quali gli aggettivi aulici «letèa» e «obliviosa»e, in struttura negativa i due «non mostra»e «non ruga», si ricollegano stilisticamente alla strofa precedente.

Le due prime strofe mostrano, per varie vie e con variatio sottile, le caratteristiche del contesto, della cornice interiore ed esteriore in cui il prodigio si sta per compiere, e che sarà il tema delle ultime due strofe del componimento. Proseguiamo adesso con la terza strofa:

Bonaccia, calura, 55

per ovunque silenzio.

L'Estate si matura

sul mio capo come un pomo

che promesso mi sia,

che cogliere io debba

con la mia mano,

che suggere io debba

con le mie labbra solo.

Perduta è ogni traccia

dell'uomo. Voce non suona,

se ascolto. Ogni duolo

umano m'abbandona.

Non ho più nome.

E sento che il mio vólto

s'indora dell'oro

meridiano,

e che la mia bionda

barba riluce

come la paglia marina;

sento che il lido rigato

con sì delicato

lavoro dall'onda

e dal vento è come

il mio palato, è come

il cavo della mia mano

ove il tatto s'affina. 
Nella terza strofa si realizza la transizione dall'ambiente al protagonista dell'evento. Al verso 58 , il «mio» richiama l'attenzione sull'io, sul soggetto protagonista dell'evento poetico. Da qui in poi, svanisce l'impersonale e tutto viene colto attraverso i sensi del poeta (cfr. v. 59: «mi», v. 60: «io», v. 61: «mia», v. 62: «io», v. 63: «mie», ecc.). La ridondanza delle espressioni personali, che qui riguardano solamente il soggetto (diversamente da quello che avverrà nella strofa conclusiva), travolgono il lettore, facendo piombare sulla scena della sua immaginazione il protagonista dell'evento: una coscienza percipiente e concepente un mondo fenomenico esterno ad essa, ma ancora per poco (siamo in una fase di transizione dell'evento panico che sarà espresso nell'ultima strofa).

Dentro quel «bonaccia» (v. 55), quel «silenzio» (v. 56) si sta per compiere, si sta per produrre (v. 57: «[...] si matura») un'epifania, un'apoteosi (cfr. v. 109), una realizzazione (v. 57 «L'Estate [...]») che al poeta è concesso di esperire (cfr. vv. 59-63: «che promesso mi sia, / che cogliere io debba / con la mia mano, / che suggere io debba / con le mie labbra solo»). Tale evento, inoltre, gli "pende", per così dire, sul «capo» (v. 58, ossia 'sulla testa'): è un'esperienza della mente.

I versi 64-68 sono il punto cardine del componimento. Siamo alla metà della terza strofa, quella della transizione dall'ambiente all'io, e quindi nel cuore di questo passaggio. Il brano indica che l'io, che fino a poco fa esperiva tutti quei fenomeni diversi, separati da sé, si sta facendo evanescente, si sta disperdendo nella totalità esperita. Non vi sono altri “io" (vv. 64-66: «Perduta è ogni traccia / dell'uomo. Voce non suona, / se ascolto. [...]»), ed anche l'io percipiente-concepente, il poeta-protagonista, sta svanendo (cfr. v. 58: «Non ho più nome»); a ciò ne consegue pertanto che: «[...] Ogni duolo / umano m’abbandona.» (vv. 66-67).

Da qui in poi comincia la simbiosi, l'unione mistica con l'ambiente, con il Tutto, prima apparente (cfr. v. 69: «E sento che [...]» e v. 75: «sento che [...]) e parziale (espressa con il ricorso alla similitudine, cfr. vv. 74: «come [...]», v. 78: «[...] è come», v. 79: «[...] è come»), per passare poi all’identificazione totale resa attraverso l'uso della metafora vera e propria (cfr. ad es. ai vv. 85-88), come accadrà nell'ultima strofa. Il volto si fa simile al Sole (cfr. vv. 60-71), la barba è come mucillagine (la "paglia marina», cfr. vv. 72-74), il palato e il palmo (cfr. v. 80: «il cavo della mia mano») sono come la spiaggia levigata dalle onde e dal vento (cfr. vv. 75-81). Il poeta si trasfigura nell'ambiente, ne viene quasi inglobato e, divenendo parte di un Tutto, si riconosce propaggine senziente di un "corpo" immenso e ne riconosce la simbiosi organica. Questa è dunque una strofa di passaggio, che descrive il momento di transizione nella metamorfosi che avverrà completamente nell'ultima. Stilisticamente, D’Annunzio esprime questa esperienza passando della similitudine (identificazione parziale) alla metafora (identificazione totale), richiamando così nella forma il processo di identificazione fra l'io e il Tutto espresso nel contenuto del componimento (Borgese 1903; Ferrari 1981a; e Burrini 2004). Giungiamo ora alla quarta e ultima strofa:

\footnotetext{
E la mia forza supina

si stampa nell'arena,

diffondesi nel mare;

e il fiume è la mia vena,

il monte è la mia fronte,

la selva è la mia pube,

la nube è il mio sudore.
} 
E io sono nel fiore

della stiancia, nella scaglia

della pina, nella bacca

del ginepro; io son nel fuco,

nella paglia marina,

in ogni cosa esigua,

in ogni cosa immane,

nella sabbia contigua,

nelle vette lontane.

Ardo, riluco.

E non ho più nome.

E l'alpi e l'isole e i golfi

e i capi e i fari e i boschi

e le foci ch'io nomai

non han più l'usato nome

che suona in labbra umane.

Non ho più nome né sorte

tra gli uomini; ma il mio nome

è Meriggio. In tutto io vivo

tacito come la Morte.

E la mia vita è divina.

La lunga preparazione emotivo-concettuale delle tre strofe precedenti trova nell'ultima la sua realizzazione. La trasfigurazione dell'io nell'ambiente e la conseguente epifania del Tutto è qui realizzata completamente. Si può dire, infatti, che l'intero componimento sia costruito secondo una logica di climax ascendente strutturale.

Ma procediamo con ordine. La strofa inizia con un'ammissione di finitezza, di totale resa: la «forza» meramente umana, propria dell'ego, dell'homo simpliciter (dice che è «mia», ossia del soggetto individuale), vinta dall'evento mistico (giace «supina», qui in un chiaro contrasto ossimorico con «la [...] forza»), si immerge nella totalità del mundus (cfr. vv. 82-84: «E la mia forza supina / si stampa nell'arena, / diffondesi nel mare»). Proprio allora però l'io, il mero ego nella sua datità individuale, scompare e si ritrova nel Tutto e nella sua intera estensione, nel contiguo e nel remoto. In ogni cosa del mondo c'è l'io e nell'io c'è il Tutto, in un gioco di specchi potenzialmente infinito. La doppia specularità dell'io e del mondo è chiaramente espressa dalla suggestiva elencatio dei versi $85-97$, che si divide in due metà che esprimono rispettivamente, il Tutto che si rispecchia nell'io (cfr. vv. 85-88) e l'io che si rispecchia nel Tutto (cfr. vv. 89-97). Come si nota dall'uso della metafora, il «come» apparente e parziale della strofa precedente è stato rimosso in favore di un'identificazione totale.

Il componimento si chiude con la presa di coscienza di quello che l'evento mistico ha portato con sé (cfr. vv. 98-99). Come il Sole, arde e riluce, e di nuovo ha perso il suo nome: tuttavia, stavolta non è il solo ad aver perso la sua "identità" umana (ossia finita, parziale): si è perduto anche il "nome delle cose". Anche l'ambiente e gli elementi che lo compongono - i quali erano stati evocati con perizia geografica nelle prime due strofe - ora si rivelano per quello che sono, nella 
loro chiarezza di fenomeno puro (cfr. vv. 100-104). Inoltre, colui che parla, con la perdita del nome e dell'identità "umane", ha perso anche il suo destino "umano" (cfr. vv. 105-106: «non ho più nome né sorte / tra gli uomini [...]»), ha perso cioè la condizione di essere finito, di essere per la morte. Con la perdita di quell'identità transitoria, illusoria, che è propria dell'io, ha acquistato una nuova identità, quella dell'eterno presente immanente: ora è il «Meriggio» (cfr. vv. 106-017), è l'hic et nunc. «Meriggio» è eternamente presente in tutte le cose (cfr. v. 107: «[...] in tutto io vivo»), ma nascostamente come lo è la loro impermanenza (cfr. v. 108: «tacito come la Morte»). Si noti che «Meriggio» e «Morte» sono personificati, pertanto sono delle entità, delle sostanze, $\mathrm{e}$ non delle condizioni o momenti transitori.

L'ultimo verso denuncia a chiare lettere la misticità, la spiritualità soggiacente al componimento. Qui si ha l'apoteosi dell'io trasfigurato nel Tutto: il trasumanare è completo. Ma è la vita ad essere divina, e non meramente il poeta-protagonista dell'evento mistico. Il concetto di «vita» dell'ultimo verso, al quale è dato l'attributo «divina», qui si collega etimologicamente al verbo usato poco sopra (cfr. vv. 107-108: «[...] in tutto io vivo / tacito come la Morte») e relativo a Meriggio; e allo stesso tempo si contrappone alla «sorte» "umana" (quindi finita e parziale) alla quale l'io di prima, non illuminato, era condannato a soggiacere, poiché imprigionato all'interno dell'orizzonte meramente umano. Quindi, la «sorte» è la condizione propria dell'essere per la morte, l'esistenza dell'individuo finito e transitorio, alla quale l'uomo è costretto (il termine 'sorte', come anche 'destino', implica l'idea di passività, di qualcosa a cui si soggiace). All'opposto, la «vita» è intesa qui come l'eternità immanente di «Meriggio», una condizione infinita e impersonale, propria invece del Sé, il quale, secondo la maggior parte delle dottrine orientali, coinciderebbe con il Principio primo, l'Assoluto - che sia esso l'Ātman-Brahman Induista o l'Anātman ('il non-sé') buddista, ossia la Śūnyatā ('la vacuità').

\section{Interpretazione}

Al termine di questa analisi si mostra chiaramente l'intento dannunziano di dare forma poetica ad una esperienza che, senza tema di esagerazione, si situa nel domino della spiritualità (ovviamente, non di carattere religioso ufficiale). Vi viene descritto un evento epifanico, gnostico, che coincide fattivamente con la trasfigurazione del soggetto cosciente nella totalità sapiente (nel senso che conosce sé stessa in quanto tale e con ciò esaurisce tutto il conoscibile, dal momento che non vi è altro da concepire oltre ad essa, assumendo in sé tutte le potenzialità dell'essere e del non essere).

Formalmente, la poesia manifesta questo evento nella sua struttura strofica, mostrando due separati oggetti - l'io cosciente e il mondo osservato da questo - che si fronteggiano nelle prime due strofe; nelle seconde due, invece, viene espressa, prima parzialmente (terza strofa), poi totalmente (quarta strofa), la fusione delle due prospettive dell'io osservatore e del non-io osservato (la natura). La concentrazione mentale dell'osservatore (si ricordi che quest'esperienza "pende" «sul [...] capo» dell'io parlante, cfr. v. 58) è talmente intensa da arrivare al punto di fondersi con l'oggetto osservato.

L'evocazione di Dante (presente nel v. 21 e chiamato addirittura «padre») non è una contingenza meramente geografica (come a dire: “D’Annunzio ha di fronte quelle due isole, si ricorda 
dei versi di Dante, e li cita"). Dante, nella tradizione italiana (e non solo), è tra i poeti sacri per eccellenza, il quale non ha solamente trattato temi spirituali nei suoi versi, ma ha anche cercato di dare forma sensibile (e quindi comunicabile) all'esperienza mistica della visione di Dio (cfr. Commedia, Par. XXXIII). Inoltre, com'è noto, nell'ultimo canto della Commedia, Dante arriva ad immergersi totalmente nella divina sostanza (cfr. Par. XXXIII, vv. 79-81: «E’ mi ricorda ch’io fui più ardito / per questo a sostener, tanto ch'i' giunsi / l'aspetto mio col valore infinito»), ossia nel Tutto (cfr. Par. XXXIII, vv. 85-89: «Nel suo profondo vidi che s'interna / legato con amore in un volume, / ciò che per l'universo si squaderna: // sustanze e accidenti e lor costume / quasi conflati insieme [...]»), e ci si rispecchia (cfr. Par. XXXIII, vv. 130-132: «dentro da sé, del suo colore stesso, / mi parve pinta de la nostra effige: / per che 'l mio viso in lei tutto era messo»), come in un certo senso accade anche nel componimento dannunziano. Quindi, l'invocazione a Dante non è contingente, né tantomeno casuale: è al contrario l'evocazione, nella mente del lettore e dentro al logos poetico, dell'architesto in cui si manifesta a pieno tale obiettivo artistico-spirituale, ossia il poetare sulla gnosi, sull'epifania dell'assoluto e sull'unione con esso. Per questo è anche chiamato «padre»: D’Annunzio ne è una progenie, così come la Meriggio lo è per la Commedia, poiché entrambi i componimenti hanno come fine ultimo dare voce, «sonare un poco» coi versi dell'esperienza sublime del riconoscersi-identificarsi con l'assoluto.

In cosa differisce però la gnosi del componimento dannunziano rispetto al canto dantesco? La differenza non è da poco. Fermo restando, per obblighi poetici, il rinvio all'Auctor, l'immagine spirituale che offre D’Annunzio è diametralmente opposta. Mentre Dante si eleva al di là della contingenza mondana (cfr. Par. XXXIII, v. 27: «più alto verso l'ultima salute»), D’Annunzio resta nel mondo e in esso trova il Tutto e l'essenza di esso (la sua radice sostanziale), alla quale si mesce assumendo l'identità di «Meriggio»: l'hic et nunc. In altre parole, per il primo, il divino è trascendente all'umano, per il secondo, è invece immanente al mondo.

Tale posizione filosofica ci conduce al nostro punto finale. Va da sé che il sostrato spirituale dannunziano si iscrive alla cultura occidentale. La sua biografia e la sua poetica sono intrise di cultura, tanto classica (in particolar modo ellenica), che cristiana (si pensi al ricorso semiotico che in vari romanzi, dall'Innocente al Fuoco, D'Annunzio fa della cultura cristiano-cattolica (Ferrara 1981b; e Di Ciaccia 2017). Va poi segnalato anche l'interesse per le tradizioni ermetico-esoteriche dell'autore (cfr. Mazza 1995). In vari punti del componimento, si nota, infatti, la conoscenza di testi gnostici, i quali innervano il loro discorso soterico sull'identificazione tra l'io

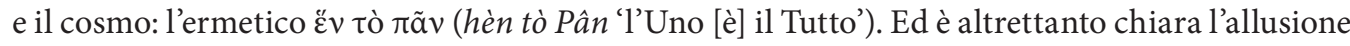
allo spirito dionisiaco nietzschiano: anche nella poesia del filosofo Il Sole declina, il meriggio è salutato come una sorta di "aurora" del dionisiaco, poiché essendo all'apogeo l'apollineo, rappresenta anche l'inizio della sua caduta inesorabile, come il Sole, che raggiunto lo zenit meridiano inizia inesorabilmente a calare (Nietzsche 2009).

Tutto questo c'è, è dietro al testo, ma c'è anche altro. C'è un'eco, infatti, più remota (nel tempo e nello spazio) che ricollega l'esperienza spirituale espressa in Meriggio alle dottrine orientali, in particolar modo alla pratica meditativa yogica. L'esperienza spirituale, l'evento mistico che la poesia esprime è estremamente simile allo stato del Samādhi (lett. 'mettere insieme', 'unire con'), nel quale il meditante sperimenta la fusione con l'oggetto della meditazione (Ferrara 1981a: 76; Ferrara 1981b: 22).

Il concetto di Samādhi è presente nell'intera tradizione spirituale indiana, lo si ritrova nello Yoga Sūtra di Patañjali, nel percorso iniziatico del Jainismo e del Sikhismo, ed anche nella tra- 
dizione buddista (il Samyak-samādhi 'retta concentrazione', ottavo ed ultimo "Nobile Sentiero" che conduce il praticante al Nirvana, la 'liberazione' dal dolore connaturato all'esistenza). Il Samādhi, inteso in un senso ampio, è dunque l'obiettivo del percorso ascetico di molte delle tradizioni religiose sorte in India, poiché è il mezzo che conduce alla gnosi dell'essenza, all'intuizione del quid ultimo delle cose, pervenendo così alla liberazione, alla Salvezza. In particolare, nella tradizione induista, la rivelazione ultima è che l'Ātman, l'essere profondo, il Sé di ogni essere, coincide con il Brahman, il dio-tutto, la deità sostanziale ed immanente a tutte le cose. Il Samādhi induista è il riconoscere che Tat tvam asi 'questa cosa [l'Ātman-Brahman] sei tu', vale a dire che l'essere individuale è l'essere supremo, il Tutto, in una identificazione totale con la deità inerente ad ogni vita e alla vita stessa. Ed è appunto quanto riconosce il D’Annunzio-Meriggio (l'essere illuminato) nel verso che chiude il componimento (cfr. v. 109), affermando chiaramente: «E la mia vita è divina» (per gli approfonditi sulla cultura spirituale indiana, si vedano, tra molti, Eliade 1999, Daniélou 2002 e Tucci 2012).

\section{Conclusione}

A fronte di quest'analisi e interpretazione di Meriggio e della discussione introduttiva dei paragrafi precedenti, si può ritenere valido ed ermeneuticamente utile il concetto di estetismo mistico come etimo semantico della poetica dannunziana.

Concludendo, si può dire quindi che lo spirituale, il divino, è parte integrante (forse addirittura centrale) nella poetica di D’Annunzio (Ferrara 1981a; e Ferrara 1981b). La spiritualità dannunziana originale trova ben presto un contrappunto nella tradizione filosofico-religiosa indiana, che porta il poeta ad approfondire sempre di più lo studio di quella cultura tanto da conformarsi ad essa o conformare essa al suo modo di sentire (Turoff 2017). Quanto detto, però, non nega l'attenzione al materiale, al fenomenico, tipica del sentire dannunziano. Anzi, in forza dell'astrazione teoretica induista, l'estetismo materiale, sempre caro al nostro, sconfina nello spirituale, senza perdere mai la sua caratteristica immanenza. Il fine poetico dannunziano, che si realizza in Alcyone e in modo particolare nell'alcionia Meriggio, è quello di compiere l'unione della materia e dello spirito, realizzando quindi l'epifania del Tutto e dunque dell'essenziale, perennemente presente in tutte le cose.

\section{Riferimenti bibliografici}

Alighieri, D. (1994). Commedia. Milano: Mondadori.

Basile, B. (1983). D’Annunzio e la lirica orientale. Lettere Italiane, 35, 2, 167-188.

Borgese, G. A. (1903). L'opera poetica di Gabriele D’Annunzio. La Nuova Antologia, 191, 106-128. (1909). Gabriele D’Annunzio. Napoli: Riccardo Ricciardi Editore.

Burrini, G. (2004). Temporalità e metamorfosi nell'«Alcyone» di D’Annunzio. Il lettore di provincia, 121, 3-15.

Costa, S. (1988). Gabriele D’Annunzio: volti e maschere di un personaggio. Firenze: Sansoni. 
Croce, B. (1904). Gabriele d'Annunzio. La Critica, 2, 85-110.

Crotti, I. (2016). Lo scrittoio imaginifico. Volti e risvolti del d'Annunzio narratore. Avellino: Edizioni Sinestesie.

Daniélou, A. (2002). Miti e dèi dell'India. Milano: BUR.

D’Annunzio, G. (1989). Il Piacere. Milano: Mondadori. (2020). Alcyone. Milano: Mondadori.

Di Ciaccia, F. (2017). L'immaginario francescano in Gabriele D’Annunzio. Roma: Aracne Editrice.

Eliade, M. (1999). Lo yoga. Immortalità e libertà. Milano: BUR.

Ferrara, M. N. (1981a). D’Annunzio e Jung. Accordo di contrari e religiosità. Quaderni del Vittoriale, 26, $65-113$

. (1981b). Religiosità e creatività in Gabriele D’Annunzio. Quaderni del Vittoriale, 28, 9-36.

Gibellini, P. (1985). Storia di «Alcyone». In Logos e Mythos. Studi su Gabriele D’Annunzio (pp. 31-84). Firenze: Olschki, 31-84.

Giusti, F. (2005), Il meriggio e la poesia. L'ora meridiana nella poesia italiana della prima metà del Novecento. Otto/Novecento: rivista quadrimestrale di critica letteraria, 29, 2, 161-170.

Leo, C. (2019). Japonaiserie nella cronaca mondana dannunziana al vaglio delle fonti francesi, In L. Curreri, L. Di Gregorio, \& F. Saenen (Eds), Tentati di morire... e di vivere: moderni barbari, esteti armati, indomabili, fratelli separati, camaleonti (pp. 70-115). Cuneo: Nerosubianco.

Mazza, A. (1995). D’Annunzio e l'occulto. Roma: Edizioni mediterranee.

Nietzsche, F. (2009) Il Sole declina. Poesia, 240.

Roncoroni, F. (2020). Introduzione a Gabriele D’Annunzio. In Alcyone (pp. 7-97). Milano: BUR.

Tucci, G. (2012). Storia della filosofia indiana. Roma-Bari: Laterza.

Turoff, B. (2017). Hinduism and the mystical aestheticism of Gabriele D’Annunzio: “Una sensualità rapita fuor de' sensi". Forum Italicum, 51, 2, 452-468.

_. (2005). Il Giappone di Gabriele D’Annunzio: Dall'estetismo al militarismo. Rivista di Studi Italiani, 22, 2, 35-57.

. (1990), Gabriele D'Annunzio and Hinduism: Spirituality, sensuality and mysticism in his novels, poetry, and "nocturnal" prose. New York: New York University (Ph.D. thesis).

— (1989). D’Annunzio e l'induismo: Testi indù e testi sull'India nella biblioteca dannunziana. Quaderni dannunziani, 5-6, 311-338.

Zollino, A. (2013), D’Annunzio fra Nietzsche e Leopardi. Evocazioni testuali e pause del tempo in "Meriggio" e "L’infinito". Nuova rivista di letteratura italiana, 16, 1-2, 109-128. 